\title{
EXTENSIONS OF DIFFERENCE SPECIALIZATIONS
}

\author{
BARBARA LANDO
}

\begin{abstract}
Maximal difference specializations and difference places are defined. Let $R$ be the domain of a difference specialization $\phi$ of a difference field $K$ and $x \in K$. Then $\phi$ can be extended to a specialization $x \rightarrow 0$ if and only if $1 \notin[x]$. This result applies to give a condition on a polynomial for the extension of a specialization to its generic zero. In a slightly different direction, a necessary and sufficient condition for the extension of a specialization to a larger difference field is given.
\end{abstract}

Introduction. Numerous examples (see [3] and below) have shown that an extension of a difference specialization may be impossible while the corresponding algebraic extension is easy to obtain. When difference extensions exist remains a question of continued interest.

This work provides conditions for the extension of a specialization to one sending a particular element to 0 (Theorems 1 and 2). It is then possible to provide, in difference algebra, conditions for extensions of specializations to extension fields (Theorems 5 and 6), which are analogous to conditions given by S. D. Morrison in differential algebra [5].

I am grateful to Richard Cohn for his helpful suggestions.

The basic definitions of difference algebra are assumed [3]. In a difference ring $R$, the difference ideal generated by a set $N$ is denoted by [N], while the perfect difference ideal is denoted $\{N\}$. Let $N^{0}=N$, let $N^{\prime}=\{a \in R \mid$ some power product of transforms of $a$ is in $N\}$ and let $N^{(k+1)}=\left[N^{(k)}\right]^{\prime}, k=1,2, \ldots$. Then $\{N\}=\cup_{k=0}^{\infty} N^{(k)}$. If $R$ is an integral domain, then any difference homomorphism of $R$ into a difference field is called a difference specialization of $R$.

1. Difference places. Let $K$ be a difference field with transforming operator $\tau$. A maximal difference specialization of $K$ is a difference homomorphism $\phi$ of a difference subring of $K$ onto a difference domain $\Lambda$, which cannot be extended to a difference homomorphism of a larger difference subring of $K$ onto a difference domain extension of $\Lambda$. It may be noted that $\Lambda$ is, in fact, a field. If $\phi(x) \neq 0$, then $\phi\left(x^{-1}\right)$ can be defined by $\phi(x)^{-1}$ in the field of quotients of $\Lambda$, but $\phi$ is maximal, so $\phi(x)^{-1} \in \Lambda$. The domain $R$ of $\phi$ is called a maximal difference ring of $K$. If $K$ is the quotient field of $R$, then $R$ is called a difference valuation ring of $K$ and $\phi$ is called a difference place of $K$. If $K$ is an inversive difference field, then any difference homomorphism of a subring of $K$ can be extended to its inversive closure in $K$, so any maximal difference ring of an inversive field is inversive.

Received by the editors January 2, 1979 and, in revised form, May 11, 1979.

AMS (MOS) subject classifications (1970). Primary 12H10; Secondary 13A15.

Key words and phrases. Difference specialization, difference place, maximal difference ring. 
Let $\phi$ be a maximal difference specialization with domain $R$ and let $M(R)$ denote the kernel of $\phi . M(R)$ is a prime reflexive difference ideal, which consists of the nonunits of $R$. Thus $R$ is a local ring with maximal ideal $M(R)$. More generally, a local difference ring is defined to be a difference ring whose nonunits form a difference ideal. Given any difference ring $R$ with prime difference ideal $P$, the local ring $R_{P}=\{r / s \mid s \notin P\}$ is a local difference ring if and only if $P$ is reflexive. This follows from the fact that $P$ is reflexive if and only if $s \notin P$ implies $s_{1} \notin P$. Consequently, the maximal ideal $M(R)$ of a local difference ring is reflexive, since in this case $R=R_{M(R)}$.

For any local difference subring of a difference field $K$, the following are equivalent: (i) $R$ is a maximal difference ring of $K$; (ii) $R$ is maximal among local difference subrings of $K$, ordered by domination; (iii) if $x \in K$ and $x \notin R$, then $1 \in\{R\{x\} M(R)\}$, the perfect difference ideal generated by $M(R)$ in $R\{x\}$. The equivalence of (iii) follows from the fact that every proper perfect difference ideal is contained in a prime reflexive difference ideal.

Let $R$ a difference valuation ring of $K$. Then the set $U$ of units of $R$ forms a subgroup of $K^{*}=K-\{0\}$ and the natural homomorphism $v: K^{*} \rightarrow K^{*} / U$ may be defined. Let $K^{*} / U$ be denoted by $\Gamma$, with the operation written as addition. Then $v$ will be called a difference valuation of $K$. Let $\Gamma^{+}=v\left(M(R)^{*}\right)$; then for $a \in \Gamma^{+},-a \notin \Gamma^{+}$. For $a$ and $b \in \Gamma$, define $a<b$ if $b-a \in \Gamma^{+}$. Then $\Gamma$ is an ordered group, but is not necessarily totally ordered. $x \in R^{*}$ if and only if $v(x)>0$ and $x \in M(R)^{*}$ if and only if $v(x)>0$.

The following example, due to R. M. Cohn, shows that there are difference valuation rings which are not valuation rings (and, thus, difference valuations which are not valuations). Let $\mathbf{C}$ be the set of complex numbers and let $a$ be transcendental over $\mathbf{C}$. Consider $\mathbf{C}(a)=\mathbf{C}\langle a\rangle$ as a difference field by defining $a_{1}=-a$. Let $\mathbf{C}\langle a\rangle\{y\}$ be a difference polynomial ring and denote the transform of $y$ by $y_{1}$. Let $P=y^{2}-y_{1}^{2}+a y^{2} y_{1}^{2}$. Then $P+P_{1}=\left(y+y_{2}\right)\left(y-y_{2}\right)\left(1+a y_{1}^{2}\right)$ and the variety $\mathfrak{R}(P)$ has 2 components, one satisfying $y+y_{2}$ and the other satisfying $y-y_{2}$. Let $x$ be a generic zero of an irreducible component, with $x_{2}=-x$. For any difference polynomial $A, x$ satisfies $A$ if and only if $x$ satisfies a first-order difference polynomial $B$ (obtained by substituting $-y$ for $y_{2}$ ), which is a multiple of $P$. It follows that $x$ specializes to 0 over $C\langle a\rangle$. This specialization can be extended to a maximal one and, hence, there is a difference valuation ring $R$ of $\mathrm{C}\langle a, x\rangle$, with $x \in M(R)$. However, $R$ is not a valuation ring: $z=x_{1} / x$ is integral over $R$ since $z$ satisfies $1-Z^{2}+a x_{1}^{2} \in R[Z]$, but $z \notin R$. If $\phi$ is the maximal specialization and $\phi(z)$ is defined, then $\phi\left(z^{2}\right)=\phi\left(1+a x_{1}^{2}\right)=1$ and $\phi\left(z z_{1}\right)=1$. But $z z_{1}=x_{1} / x \cdot x_{2} / x_{1}=-1$. Thus, $z$ is not in the domain of $\phi$.

2. Extensions of a specialization to an element. The following criterion provides a tool in specialization problems, as well as in the development of the theory of difference places.

TheOREM 1. Let $R$ be a local difference subring of a difference field $K$ and let $x \in K$. The homomorphism $\phi: R \rightarrow R / M(R)$ extends to one sending $x$ to 0 if and only if $1 \notin[x]$ in $R\{x\}$. 
Proof. If $\phi$ extends to a homomorphism $\phi^{\prime}$ of $R\{x\}$ with $\phi^{\prime}(x)=0$, then $[x] \subset \operatorname{Ker} \phi^{\prime}$. Therefore $1 \notin[x]$.

For the converse, let $N$ denote the difference ideal generated by $M(R)$ and $x$ in $R\{x\}$. Then $N=R\{x\} M(R)+[x]=M(R)+[x]$. If $\phi$ cannot be extended to a homomorphism of $R\{x\}$ sending $x$ to 0 , then $1 \in\{N\}$. It will be shown by an inductive argument that if there is $c \in\{N\}$ with $c=u+z, u \in U=R-M(R)$, $z \in[x]$, then $1 \in[x]$. Since $1 \in\{N\}$ and 1 is of this form (with $z=0$ ), the proof will be complete.

$\{N\}=\cup_{k=0}^{\infty} N^{(k)}$, so $c \in\left[N^{(k)}\right]$ for some $k$. If $k=0$, then $c \in N=M(R)+$ $[x]$. Thus $c=u+z=m+w$, where $m \in M(R)$ and $w \in[x]$. Since $u \notin M(R)$, $u-m \notin M(R)$ and $(u-m)^{-1} \in R$. Then $1=(u-m)^{-1}(u-m)=(u-$ $m)^{-1}(w-z) \in[x]$. If $k=n+1$, then $c=\sum_{i=1}^{p} f_{i} q_{i}$ where $f_{i} \in R\{x\}$ and $q_{i} \in$ $N^{(n+1)}$. For each $i, f_{i}=r_{i}+z_{i}$ and $q_{i}=s_{i}+y_{i}$, where $r_{i}$ and $s_{i}$ are in $R$ and $z_{i}$ and $y_{i}$ are in [x]. So $\sum_{i=1}^{p} f_{i} q_{i}=\sum_{i=1}^{p}\left(r_{i}+z_{i}\right)\left(s_{i}+y_{i}\right)=t+w$, where $t=\sum_{i=1}^{p} r_{i} s_{i}$ and $w \in[x]$. Thus, $c=u+z=t+w$. If $t \in M(R)$, then, as above, $(u-t)^{-1} \in R$ and $1 \in[x]$. Otherwise, $s_{i} \notin M(R)$ for some $i$. For this $i$, denote $q_{i}=s_{i}+y_{i}$ simply by $q=s+y$. Since $q \in N^{(n+1)}$, there is a product $\pi(q)=(s+y)^{p_{0}}(\tau s+$ $\tau y)^{p_{1}} \ldots\left(\tau^{j_{s}}+\tau^{j} y\right)^{p_{j}} \in\left[N^{(n)}\right]$. But $\pi(q)=\bar{u}+\bar{z}$, where $\bar{u}=s^{p_{o}}(\tau s)^{p_{1}} \cdots\left(\tau^{j}\right)^{p_{j}}$ and $\bar{z} \in[x]$. Since $s \notin M(R)$ and $M(R)$ is prime and reflexive, $\bar{u} \in R-M(R)$. Therefore, by induction on $n, 1 \in[x]$.

Corollary. Let $R$ be a maximal difference ring of $K$, and let $x \in K$. Then $x \in M(R)$ if and only if $1 \notin[x]$.

Let $K\{y\}$ be a difference polynomial ring with transform $\tau$ and let $\phi$ be a difference specialization of $K$ with domain $R$. Let $g(y) \in K\{y\}$ and let $x$ be a generic zero of a component of $\mathfrak{N}(g)$. The specialization of the coefficients of $g$ to 0 does not guarantee that $\phi$ can be extended to a specialization of $x$ to 0 . R. M. Cohn has noted that even if $g(y)$ is of the form $y \tau y+b$, this may not be possible: let $b$ be a nonzero solution of the polynomial $Q$ on p. 332 of [3]. However, a sufficient condition for such an extension can be given.

Let $R$ be a difference subring of a difference field $K$. Let $g(y)$ be a difference polynomial in $R\{y\}$. Let $\{g\}_{R}$ and $\{g\}_{K}$ be the perfect difference ideals generated by $g$ in $R\{y\}$ and $K\{y\}$, respectively. The term of the polynomial which has no power of $y$ or its transforms is called the constant term.

TheOREM 2. Let $g(y) \in R\{y\}$ have constant term $b \in R$. Let $\{g\}_{K}$ be prime and let $x$ be a generic zero of $\{g\}_{K}$. Let $\phi: R \rightarrow \Lambda$ be a difference specialization of $K$ with $\phi(b)=0$. $\phi$ can be extended to $R\{x\}$ with $\phi(x)=0$ if $\{g\}_{K} \cap R\{y\}=\{g\}_{R}$.

Proof. It can be shown by induction that if $f(y) \in\{g\}_{R}$ and has constant term $c$, then $\phi(c)=0 .\{g\}_{R}=\cup_{k=0}^{\infty}[g]^{(k)}$, so $f \in[g]^{(k)}$ for some $k$. If $k=0, f(y)=$ $\sum_{i=1}^{p} f_{i}(y) \tau^{i} g(y), f_{i} \in R\{y\}$. Comparison of constant terms yields $c=\sum_{1}^{p} r_{i} \tau^{i} b$, $r_{i} \in R$, and hence $\phi(c)=0$. If $k=n+1$, then $f(y)=\Sigma_{1}^{p} f_{i}(y) q_{i}(y)$, where $f_{i} \in$ $R\{y\}$ and a power product $\pi_{i}\left(q_{i}\right) \in[g]^{(n)}$. If $q_{i}$ has constant term $a_{i}$, then $\pi_{i}\left(q_{i}\right)$ has constant term $\pi_{i}\left(a_{i}\right)$ and by induction, $\phi\left(\pi_{i}\left(a_{i}\right)\right)=0$. Hence $\phi\left(a_{i}\right)=0$ and $\phi(c)=$ $\phi\left(\sum r_{i} a_{i}\right)=0$. Consequently, if $f(y) \in\{g\}_{R}$, its constant term $\neq 1$. 
By extending $\phi$ in $K$, one may assume that $R$ is a local difference subring of $K$ and hence of $K\{x\}$, with $\{g\}_{K} \cap R\{y\}=\{g\}_{R}$. (For, if $P=\operatorname{Ker} \phi$ and $R$ is replaced by $S=R_{P}$, it follows that $\{g\}_{K} \cap S\{y\}=\{g\}_{s}$.) By Theorem 1 , if $\phi$ does not extend to $x \rightarrow 0$, then $1 \in[x]$ in $R\{x\}$, i.e. $1=\Sigma b_{i}(x) \tau^{i}(x), b_{i} \in R\{x\}$. Then $f(y)=1-\Sigma b_{i}(y) \tau^{i}(y) \in\{g\}_{K} \cap R\{y\}$. Since $f$ has constant term $1, f \notin$ $\{g\}_{R}$. Hence, $\{g\}_{K} \cap R\{y\} \neq\{g\}_{R}$.

The corollary to Theorem 1 applies to maximal difference rings, yielding the following development.

Proposition 1. Let $R$ and $S$ be maximal difference rings of $K$ with $R \subset S$. Then $M(S) \subset M(R)$.

Proof. If $x \in M(S)$, then $1 \notin[x] \cdot S\{x\}$. But, since $R \subset S, 1 \notin[x] \cdot R\{x\}$. Hence $x \in M(R)$.

It follows that if $R$ and $S$ are maximal difference rings of $K$ with $R \subset S$, then every ideal of $S$ is an ideal of $R$.

Proposition 2. Let $R$ be a maximal difference ring of $K$, and let $P$ be a prime reflexive difference ideal of $R$. Then there is a maximal difference ring $S$ of $K$ such that $R \subset S$ and $M(S)=P$.

Proof. Let $S$ be a maximal local difference ring of $K$, dominating $R_{P}$. By Proposition 1, $M(S) \subset M(R)$. Hence $M(S)=M(S) \cap R=\left(M(S) \cap R_{P}\right) \cap R=$ $P \cdot R_{P} \cap R=P$.

The next proposition follows from this with a proof similar to that given in the differential case in [5, Proposition 3].

Proposition 3. Let $R$ be a maximal difference ring of $K$. Then the prime reflexive difference ideals of $R$ are linearly ordered by inclusion.

As a consequence, in a maximal difference ring, every perfect difference ideal is prime. The difference rank of a difference valuation ring is defined to be the number of prime reflexive ideals in the ring.

Proposition 4. Let $S$ be a maximal difference ring of $K$ with specialization $\phi$ : $S \rightarrow \Lambda$. If $R$ is a maximal difference ring of $K$ with $R \subset S$, then $\phi(R)$ is a maximal difference ring of $\Lambda$.

Proof. Let $\psi: R \rightarrow \Omega$ be a maximal specialization of $K$ with domain $R$. Since $\operatorname{Ker} \phi=M(S) \subset M(R)=\operatorname{Ker} \psi, \theta: \phi(R) \rightarrow \Omega$ may be defined by $\theta(\phi(r))=\psi(r)$, with $\operatorname{Ker} \theta=\phi(M(R))$. Let $x \in \Lambda$; if $x \notin \phi(R)$, there is $s \in S, s \notin R$, such that $\phi(s)=x$. Since $\psi$ is maximal, $1 \in\{R\{s\} \cdot M(R)\}$ and thus, $1 \in\{\phi(R)\{x\}$. $\operatorname{Ker} \theta\}$. Therefore $\theta$ cannot be extended to $x$.

3. Extensions of a place to an extension field. If $\phi$ is a difference place of $K$ and $L$ is a difference field extension of $K$, there arises the question of whether $\phi$ can be extended to a difference place of $L$. A necessary and sufficient condition is provided in the corollary below. The development is analogous to that of the differential case [5]. 
TheORem 3. Let $R_{0}$ be a difference subring of $K$ with prime reflexive ideals $P$ and $Q, P \subset Q$ and let $S$ be a proper, maximal difference ring of $K$ with $R_{0} \subset S$ and $M(S) \cap R_{0}=P$. Then there is a proper maximal difference ring $R$ with $R_{0} \subset R$ and $M(R) \cap R_{0}=Q$. Furthermore, if $S$ is a difference valuation ring of $K$ then $R$ is also.

Proof. Similar to Theorem 1 [5].

COROLlARY 1. Let $R_{0}$ be a local difference subring of a difference field $K$. Let $L$ be $a$ difference field extension of $K$ and let $S$ be proper maximal difference ring (valuation ring) of $L$ containing $R_{0}$. Then there is a proper maximal difference ring (valuation ring) $R$ of $L$ dominating $R_{0}$.

The existence of $S$ in the corollary is equivalent to the condition that $L$ have a subring $S_{0}, R_{0} \subset S_{0}$, which contains a proper nonzero prime reflexive difference ideal. That the condition does not always hold is easily seen. For example, if $\mathbf{Q}\{b\}$ is a difference ring with $b$ transcendental over the rationals $\mathbf{Q}$ and $\tau b=b$, the difference specialization $b \rightarrow 1$ does not extend to a difference place of $\mathbf{Q}\langle a\rangle$, where $a^{2}=b$ and $\tau a=-a$. (See [3, Chapter 7, Example 3].) However, the condition does hold in the situations of Theorems 5 and 6 . Theorem 4 is needed for these results.

THeOREM 4. Let $R$ be a difference integral domain with quotient field $K$. If $K\left\langle a_{1}, \ldots, a_{n}\right\rangle$ is a difference field extension of $K$ which is a primary extension, then there is $u \in R, u \neq 0$, such that any specialization $\phi$ of $R$ with $\phi u \neq 0$ can be extended to $R\left\{a_{1}, \ldots, a_{n}\right\}$.

This theorem is a slight modification of part of Theorem IV of [3, Chapter 7] (where $R=F\left\{b_{1}, \ldots, b_{m}\right\}, F$ is a difference field and the specializations are over $F)$. The generalization to an arbitrary domain $R$ can be obtained by using Proposition 9, Chapter 0 of [4] in the lemmas preceding the theorem.

THEOREM 5. Let $R_{0}$ be a local difference ring with quotient field $K$ and no minimal nonzero prime reflexive difference ideals. Let $L$ be a primary, finitely-generated extension of $K, L=K\left\langle a_{1}, \ldots, a_{n}\right\rangle$. Then there is a difference valuation ring $R$ of $L$ dominating $\boldsymbol{R}_{\mathbf{0}}$.

The proof is analogous to that in the differential case (see Theorem 2 of [5]), following here from Theorems 3 and 4.

THEOREM 6. Let $R_{0}$ be a local difference ring with quotient field $K$. Let $b$ be transformally independent over $K$ and let $L=K\left\langle b, a_{1}, \ldots, a_{n}\right\rangle$ be a primary extension of $K\langle b\rangle$. Then there is a difference valuation ring of $L$ dominating $R_{0}$.

Proof. In this case, the ideals $P_{k}=\left\{b-\tau^{k} b\right\}, k=1,2, \ldots$, provide a descending chain of prime reflexive ideals in $R\{b\}$, so that if $u \in R\{b\}, u \neq 0$, then 
$u \notin P_{k}$ for some $k$. Then Theorem 4 yields a prime reflexive difference ideal in $R\left\{b, a_{1}, \ldots, a_{n}\right\}$ and the proof proceeds as in [5].

\section{REFERENCES}

1. P. Blum, Complete models of differential fields, Trans. Amer. Math. Soc. 137 (1969), 309-325.

2. ___ Extending differential specializations, Proc. Amer. Math. Soc. 24 (1970), 471-474.

3. R. M. Cohn, Difference algebra, Interscience, New York, 1965.

4. E. R. Kolchin, Differential algebra and algebraic groups, Academic Press, New York, 1973.

5. S. D. Morrison, Extensions of differential places, Amer. J. Math. 100 (1978), 245-261.

Department of Mathematics, University of Alasta, Fairbantss, Alasta 99701 